\title{
A influência da obesidade na Covid-19 grave
}

The influence of obesity on severe Covid-19

La influencia de la obesidad en el Covid-19 severo

Emanuelle Paiva de Vasconcelos Dantas

ORCID: https://orcid.org/0000-0001-7752-0416 Centro Universitário Uninassau Aliança Redenção, Brasil E-mail: emanuellepaiva@yahoo.com.br

Vitor Kauê de Melo Alves

ORCID: https://orcid.org/0000-0002-7283-0850 Universidade Estadual do Piauí, Brasil

E-mail: vitorkaue_@hotmail.com

Alaíde Silva Lemos

ORCID: https://orcid.org/0000-0003-4121-6724 Centro Universitário Uninovafapi, Brasil

E-mail: laid_mel13@hotmail.com

Isadora Alencar da Silva Andrade

ORCID: https://orcid.org/0000-0002-0195-4514 Centro Universitário Uninovafapi, Brasil E-mail: Isadoraandrade733@gmail.com

Luciana Alencar Fialho Bringel ORCID: https://orcid.org/0000-0001-6004-5727 Universidade Federal do Maranhão, Brasil E-mail: luciana_bringel@ hotmail.com

Yaskara Raissa de Pádua Sampaio ORCID: https://orcid.org/0000-0001-5983-2387 Centro Universitário Uninovafapi, Brasil E-mail: Yaskararaissa@gmail.com

Sérgio Henrique Assunção Lacerda Borges ORCID: https://orcid.org/0000-0002-8553-7223 Centro Universitário Uninovafapi, Brasil E-mail: seergiol@hotmail.com

Cristiane de Souza Pantoja ORCID: https://orcid.org/0000-0002-9681-402X Universidade da Amazônia, Brasil

E-mail: contatocristianesouza@ outlook.com

Fernanda Nascimento Silva

ORCID: https://orcid.org/0000-0001-7210-1140 Universidade Estadual do Piauí, Brasil

E-mail: enf.fernandans@gmail.com

Taynara de Sousa Rego Mendes ORCID: https://orcid.org/0000-0003-3321-2408 Centro Universitário Santo Agostinho, Brasil E-mail: taynara.srm@gmail.com

Karolinne Adrião de Oliveira

ORCID: https://orcid.org/0000-0002-9134-4292

Centro Universitário Maurício de Nassau, Brasil

E-mail: karoladriao_oliveira@hotmail.com

Matheus Pedrosa de Oliveira

ORCID: https://orcid.org/0000-0003-4003-3184

Universidade Federal do Delta do Parnaíba, Brasil

E-mail: matheus_pedrosasete@hotmail.com

Jacyra Pereira Oliveira

ORCID: https://orcid.org/0000-0003-2177-0875

Universidade Federal do Pará, Brasil

E-mail: teclog.jacyra@gmail.com 


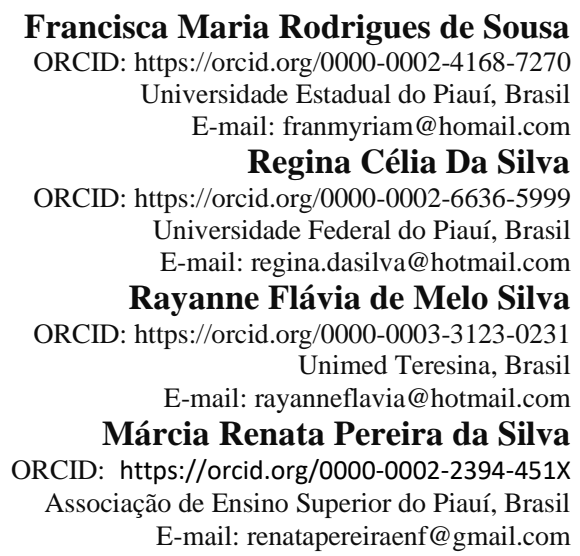

\title{
Resumo
}

A obesidade é definida como o excesso de acúmulo de tecido adiposo, e sua quantidade apresentam danos à saúde. Além de ser um fator agravante para múltiplas comorbidades, também pode levar a graves doenças metabólicas e respiratórias. Sua etiologia é muito complexa e possui características multifatoriais. O trabalho tem por objetivo expor através da análise de artigos científicos a influência da obesidade na Covid-19 grave. Trata-se de uma revisão bibliográfica de caráter qualitativo que se baseia na produção científica a partir de estudos já publicados entre os anos de 2019 a 2021. Dados internacionais preocupantes mostram que a frequência de obesidade é muito alta entre pacientes gravemente enfermos que recebem tratamento intensivo de SARS-CoV-2. No Brasil, a obesidade é a principal comorbidade associada ao óbito em menores de 60 anos. Pessoas obesas têm quatro vezes mais risco de lesões e morte devido ao COVID-19. É necessária a realização de pesquisas utilizando protocolos clínicos e epidemiológicos para determinar a relação entre sobrepeso, obesidade e aumento do risco de complicações do COVID-19, a fim de gerar e estabelecer um entendimento mais profundo e melhor dessa associação, incluindo o entendimento das complicações concomitantes de pacientes com sobrepeso.

Palavras-chave: Covid-19; Infecção por SARS-CoV-2; Fator de risco; Obesidade.

\begin{abstract}
Obesity is defined as the excess accumulation of adipose tissue, and its quantity is harmful to health. In addition to being an aggravating factor for multiple comorbidities, it can also lead to serious metabolic and respiratory diseases. Its etiology is very complex and has multifactorial characteristics. The work aims to expose through the analysis of scientific articles the influence of obesity in severe Covid-19. This is a literature review of a qualitative nature based on scientific production based on studies already published between the years 2019 to 2021. Concerning international data show that the frequency of obesity is very high among critically ill patients receiving intensive care of SARS CoV-2. In Brazil, obesity is the main comorbidity associated with death in children under 60 years of age. Obese people are four times more at risk of injury and death from Covid-19. Research using clinical and epidemiological protocols is needed to determine the relationship between overweight, obesity and increased risk of complications from Covid-19, in order to generate and establish a deeper and better understanding of this association, including an understanding of complications of overweight patients.
\end{abstract}

Keywords: Covid-19; SARS-CoV-2 infection; Risk factor; Obesity.

\section{Resumen}

La obesidad se define como la acumulación excesiva de tejido adiposo y su cantidad es perjudicial para la salud. Además de ser un factor agravante de múltiples comorbilidades, también puede conducir a enfermedades metabólicas y respiratorias graves. Su etiología es muy compleja y tiene características multifactoriales. El trabajo tiene como objetivo exponer a través del análisis de artículos científicos la influencia de la obesidad en el Covid-19 severo. Se trata de una revisión de la literatura de carácter cualitativo basada en la producción científica basada en estudios ya publicados entre los años 2019 a 2021. A nivel internacional, los datos muestran que la frecuencia de obesidad es muy alta entre los pacientes críticamente enfermos que reciben cuidados intensivos de SARS-CoV-2. En Brasil, la obesidad es la principal comorbilidad asociada a la muerte en menores de 60 años. Las personas obesas tienen cuatro veces más riesgo de lesiones y muerte por Covid-19. Se necesitan investigaciones que utilicen protocolos clínicos y epidemiológicos para determinar la relación entre el sobrepeso, la obesidad y el aumento del riesgo de complicaciones por Covid-19, con el fin de generar y establecer una comprensión más profunda y mejor de esta asociación, incluida la comprensión de las complicaciones de los pacientes con sobrepeso.

Palabras clave: Covid-19; Infección por SARS-CoV-2; Factor de riesgo; Obesidad. 


\section{Introdução}

De acordo com a Organização Mundial da Saúde (OMS), entre os anos de 1975 a 2016, a prevalência global da obesidade triplicou e continuou a aumentar em países de baixa e média renda, deixando de ser um problema apenas nos países mais ricos. Em 2016, mais de 1,9 bilhões de adultos apresentavam sobrepeso e mais de 650 milhões eram obesos (Pitanga $e t$ al., 2020).

A obesidade é definida como o excesso de acúmulo de tecido adiposo, e sua quantidade apresentam danos à saúde. Além de ser um fator agravante para múltiplas comorbidades, também pode levar a graves doenças metabólicas e respiratórias. Sua etiologia é muito complexa e possui características multifatoriais (Martelleto et al., 2021).

Uma das características da obesidade é a inflamação crônica de baixo grau associada a várias complicações. A resposta inflamatória é ativada na fase inicial do aumento do tecido adiposo em pacientes obesos crônicos, levando a alterações permanentes no sistema imunológico a um fenótipo pró-inflamatório. Esses mecanismos envolvem a ativação constante do sistema imunológico inato, causando danos aos órgãos responsáveis por manter a homeostase corporal (Schuh, 2021).

No Brasil, a prevalência de obesidade é motivo de preocupação, principalmente na situação atual causada pela pandemia de Covid-19, considerando que mais da metade da população sofre com sobrepeso (56,7\%). Também é importante ressaltar que o aumento da prevalência é ainda maior na faixa etária entre 18 e 24 anos. Esses dados ajudam a aumentar as complicações e agravamento dos sintomas em pessoas infectadas com menos de 60 anos de idade. Segundo relatório do Ministério da Saúde, em São Paulo, Santa Catarina, Pernambuco, Bahia, Espírito Santo e Rio Grande do Norte o número de óbitos é alarmante (De Figueiredo et al., 2020).

A COVID-19 foi declarada uma pandemia global em março de 2020. É considerada uma morbidade de alta incidência de transmissão pelo vírus de RNA de fita positiva SARS-CoV-2, resultando em efeitos nocivos no sistema respiratório e intestinal de alguns hospedeiros, causando sintomas que variam de leves a fatais. Essa infecção já afetou mais de 3.000.000 de pessoas e matou mais de 200.000 pessoas em todo o mundo. Preditores de resultados emergentes incluem idosos, devido ao envelhecimento imunológico, e homens, bem como pessoas com anormalidades metabólicas, como hipertensão, diabetes e obesidade (Almeida et al., 2020).

A relação entre obesidade e doenças causadas por vírus tem sido estudada há vários anos. Durante a epidemia de H1N1, esse campo atraiu especial interesse, pois foi observado que pacientes obesos apresentavam maior risco de contrair a infecção, maior tempo de permanência na UTI e altas taxas de mortalidade. Esse fato já foi confirmado até mesmo em crianças obesas, cuja resposta imunológica ao vírus influenza é prejudicada, principalmente a resposta imune celular, e a resposta vacinal de indivíduos obesos também é insuficiente (Nogueira de Almeida et al., 2020).

Os sintomas da infecção por SARS- CoV-2 podem variar de assintomática a síndrome do desconforto respiratório agudo e disfunção de múltiplos órgãos. De acordo com a OMS, a maioria dos pacientes com Covid-19 (cerca de 80\%) pode ser Assintomáticos, cerca de $20 \%$ dos casos podem necessitar de hospitalização por dispneia e, nestes casos, cerca de $5 \%$ podem necessitar de suporte de tratamento para insuficiência respiratória (suporte ventilatório) (De Figueiredo et al., 2020).

Alguns dados atuais indicam que a obesidade pode estar associada a resultados desfavoráveis, principalmente quando associada a outras doenças, como envolvimento de órgãos vitais, distúrbios endócrinos e metabólicos e aumento das respostas imunoinflamatórias. Todas essas alterações parecem estar relacionadas ao acúmulo excessivo de gordura nos órgãos internos ou áreas ectópicas, e não no subcutâneo. Pessoas com IMC discretamente mais alto, principalmente na área subcutânea, são frequentemente chamadas de "obesos metabolicamente saudáveis" e, teoricamente, podem estar mais protegidos (Giacaglia, 2020).

O trabalho tem por objetivo expor através da análise de artigos científicos a influência da obesidade na Covid-19 grave. 


\section{Metodologia}

Trata-se de uma revisão bibliográfica de caráter qualitativo que se baseia na produção científica a partir de estudos já publicados. A busca pelos textos foi realizada a partir das seguintes palavras-chaves indexadas no DECS (Descritores em Ciências da Saúde): "Covid-19"; "Infecção por SARS-CoV-2"; "Fator de risco" e "Obesidade"; na plataforma Scientific Electronic Library Online (SCIELO) e Biblioteca Virtual em Saúde (BVS).

Os critérios de inclusão foram pesquisas científicas publicadas de 2019 a 2021, artigos completos disponíveis na íntegra, teses e dissertações publicados no idioma português, inglês e espanhol, que atendiam ao problema da pesquisa: Qual a influência da obesidade na Covid-19 grave? Os critérios de exclusão foram trabalhos científicos com apenas resumos disponíveis, publicações duplicadas, artigos de relato de experiência, reflexivo, editoriais, comentários e cartas ao editor.

A partir do problema de pesquisa foram selecionados artigos publicados em periódicos nacionais e internacionais escolhidos a partir de levantamento realizado por meios dos descritores na biblioteca virtual Scientific Electronic Library Online, Biblioteca Virtual em Saúde (BVS) e dissertações e teses.

Os artigos foram selecionados para leitura e foram incluídos na análise artigos que se enquadravam no tema proposto do trabalho.

\section{Resultados e Discussão}

A Figura 1 ilustra os resultados deste trabalho que foram obtidos através da análise quantitativa de publicações sobre a influência da obesidade na Covid-19 grave nas bases de dados Scientific Electronic Library Online e Biblioteca Virtual em Saúde (BVS).

Figura 1. Fluxograma que apresenta o processo de seleção das publicações sobre a influência da obesidade na Covid-19 grave, Teresina, Brasil, 2021.

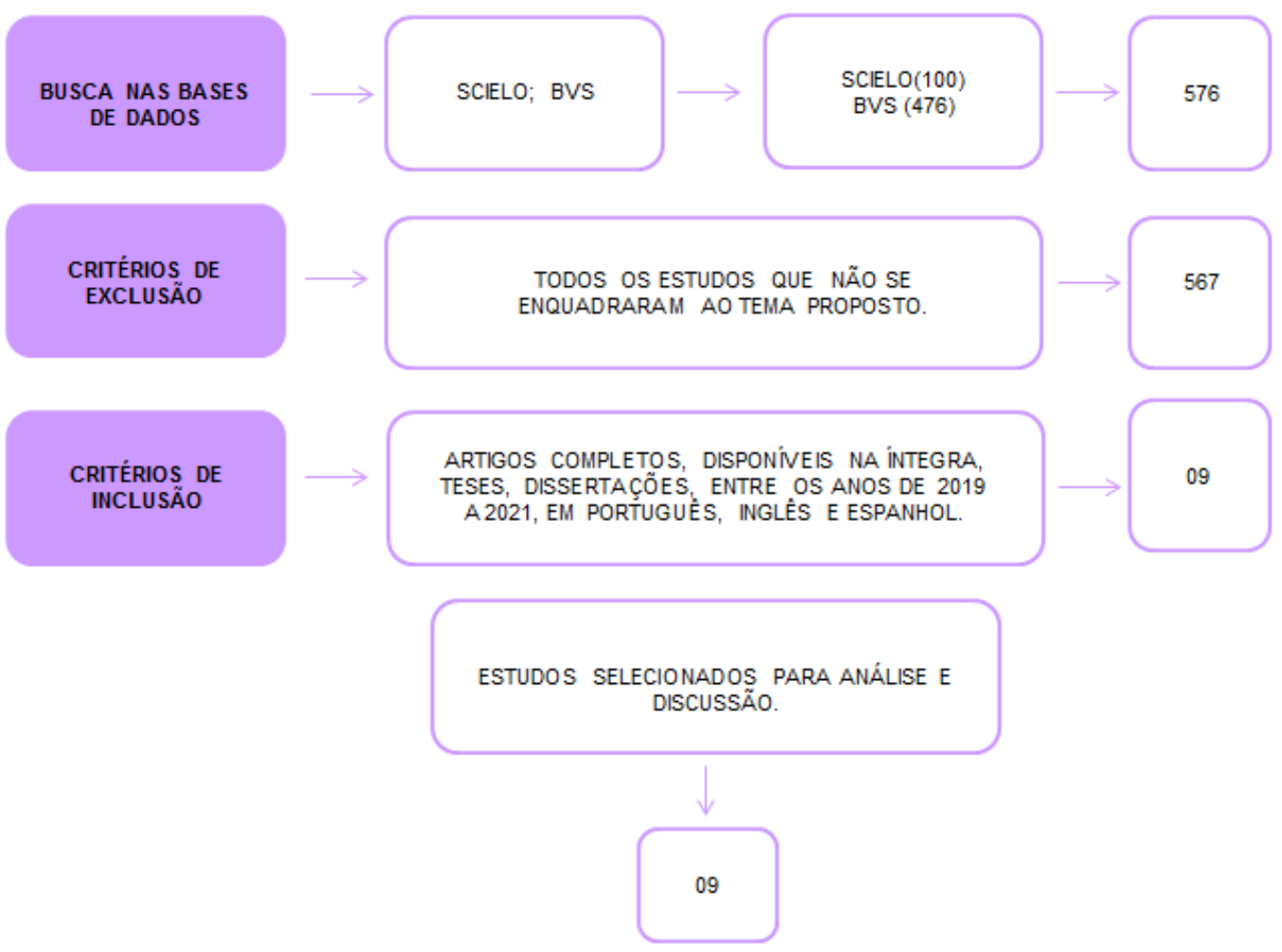

Fonte: Mota et al., (2021). 
Os resultados deste trabalho foram obtidos através da análise quantitativa de publicações sobre a influência da obesidade na Covid-19 grave, encontrados nas bases de dados Scientific Electronic Library Online e Biblioteca Virtual em Saúde obtendo 100 artigos na Scielo e 476 artigos na BVS. Foram excluídos 96 artigos da Scielo e 471 da BVS por não se enquadrarem no tema proposto. Foram incluídos 04 artigos da Scielo e 05 artigos da BVS entre os anos de 2019 a 2021 para a análise e discussão.

De acordo com a pesquisa realizada nas bases de dados, foi possivel listar os principais títulos e objetivos dos estudos publicados na literatura, utilizados neste trabalho conforme o Quadro 1.

Quadro 1. Principais títulos e objetivos dos artigos utilizados na pesquisa, Teresina, Brasil, 2021.

\begin{tabular}{|c|c|c|}
\hline ANO DE PUBLICAÇÃO & TÍTULO & OBJETIVO \\
\hline $\begin{array}{l}\text { Nogueira de Almeida et al., } \\
2020 \text {. }\end{array}$ & $\begin{array}{l}\text { COVID-19 e obesidade na infância e } \\
\text { adolescência: uma revisão clínica. }\end{array}$ & $\begin{array}{l}\text { Identificar os fatores que contribuem para o aumento } \\
\text { da suscetibilidade e gravidade da COVID- } 19 \text { em } \\
\text { crianças e adolescentes obesos e suas consequências } \\
\text { para a saúde. }\end{array}$ \\
\hline De Figueiredo et al., 2020. & $\begin{array}{l}\text { O impacto do excesso de peso nas } \\
\text { complicações clínicas causadas pela COVID- } \\
\text { 19: Uma revisão sistemática. }\end{array}$ & $\begin{array}{l}\text { O objetivo foi avaliar e demonstrar os principais } \\
\text { impactos do excesso de peso nas complicações e } \\
\text { desfechos clínicos ocasionados pelo SARS-CoV-2. }\end{array}$ \\
\hline Almeida et al., 2020. & $\begin{array}{l}\text { Preditores de riscos advindos da obesidade } \\
\text { que repercutem em mau prognóstico em } \\
\text { pacientes infectados por Sars-CoV-2. }\end{array}$ & $\begin{array}{l}\text { O presente estudo teve como objetivo investigar os } \\
\text { fatores de risco relacionados à obesidade que } \\
\text { reverberam para o mau prognóstico de pacientes } \\
\text { obesos infectados por Sars-Cov- } 2 \text {. }\end{array}$ \\
\hline Pitanga et al., 2020. & $\begin{array}{l}\text { Inatividade física, obesidade e COVID-19: } \\
\text { perspectivas entre múltiplas pandemias. }\end{array}$ & $\begin{array}{l}\text { O presente ensaio teórico tem como objetivo analisar } \\
\text { a interface entre as pandemias de inatividade física, } \\
\text { obesidade e COVID- } 19 \text {. }\end{array}$ \\
\hline Martelleto et al., 2021. & $\begin{array}{l}\text { Principais fatores de risco apresentados por } \\
\text { pacientes obesos acometidos de COVID-19: } \\
\text { uma breve revisão. }\end{array}$ & $\begin{array}{l}\text { Esse artigo busca abordar e discutir os mecanismos } \\
\text { fisiopatológicos inerentes aos principais fatores de } \\
\text { risco que levam ao pior prognóstico observado em } \\
\text { pacientes com COVID-19. }\end{array}$ \\
\hline Bolsoni-Lopes et al., 2021. & $\begin{array}{l}\text { Obesidade e a Covid-19: uma reflexão sobre a } \\
\text { relação entre as pandemias. }\end{array}$ & $\begin{array}{l}\text { Realizar uma análise reflexiva sobre a relação da } \\
\text { obesidade como fator de risco para o agravamento } \\
\text { dos quadros de COVID- } 19 \text {. }\end{array}$ \\
\hline Silva et al., 2021. & $\begin{array}{l}\text { Obesidade como fator agravante da COVID- } \\
19 \text { em adultos hospitalizados: revisão } \\
\text { integrativa. }\end{array}$ & $\begin{array}{l}\text { Identifi car, na literatura científi ca, a relação da } \\
\text { obesidade como fator de risco agravante para a } \\
\text { morbidade por COVID- } 19 \text {. }\end{array}$ \\
\hline Christinelli et al., 2021. & $\begin{array}{l}\text { Percepções de adultos com obesidade sobre } \\
\text { monitoramento remoto multiprofissional no } \\
\text { início da pandemia de COVID- } 19 \text {. }\end{array}$ & $\begin{array}{l}\text { Compreender a percepção de adultos com obesidade } \\
\text { sobre o monitoramento remoto no início da pandemia } \\
\text { de COVID-19. }\end{array}$ \\
\hline Costa et al., 2020. & $\begin{array}{l}\text { Obesidade infantil e quarentena: crianças } \\
\text { obesas possuem maior risco para a COVID- } \\
19 \text { ? }\end{array}$ & $\begin{array}{l}\text { Dissertar sobre a correlação entre a infecção pelo } \\
\text { vírus SARS-CoV-2 e a obesidade no grupo pediátrico } \\
\text { e suas possíveis consequências e fatores de } \\
\text { gravidades, possibilitando, assim, maior abrangência } \\
\text { em relação ao tema para construção de novas } \\
\text { propostas. }\end{array}$ \\
\hline
\end{tabular}

Fonte: SCIELO e BVS.

Desta forma, foi possível listar os principais resultados e conclusões dos estudos publicados na literatura, conforme o Quadro 2. 
Quadro 2. Resultados e conclusões dos artigos utilizados, Teresina, Brasil, 2021.

\begin{tabular}{|c|c|c|}
\hline PUBLICAÇÃO & RESULTADOS & CONCLUSÕES \\
\hline $\begin{array}{l}\text { Nogueira de Almeida et al., } \\
\qquad 2020 .\end{array}$ & $\begin{array}{l}\text { A obesidade é uma comorbidade altamente prevalente em } \\
\text { casos graves de COVID-19 em crianças e adolescentes; o } \\
\text { isolamento social pode levar ao aumento do acúmulo de } \\
\text { gordura. Tecido adiposo excessivo, déficit de massa magra, } \\
\text { resistência à insulina, dislipidemia, hipertensão, níveis elevados } \\
\text { de citocinas pró-inflamatórias e baixa ingestão de nutrientes } \\
\text { essenciais são fatores que comprometem o funcionamento de } \\
\text { órgãos e sistemas em indivíduos obesos. }\end{array}$ & $\begin{array}{l}\text { A necessidade de isolamento social pode ter } \\
\text { como efeito causar ou agravar a obesidade e } \\
\text { suas comorbidades, e o pediatra deve estar } \\
\text { atento a essa questão. }\end{array}$ \\
\hline De Figueiredo et al., 2020. & $\begin{array}{l}\text { O excesso de peso pode atuar desregulando as respostas } \\
\text { linfoides e mieloides, as quais irão alterar o sistema } \\
\text { imunológico e estender respostas inflamatórias, contribuindo } \\
\text { para a maior proliferação de infecções virais, como ocorre na } \\
\text { pandemia do COVID-19. }\end{array}$ & $\begin{array}{l}\text { Portanto, nota-se que o quadro de obesidade e } \\
\text { excesso de peso atuam diretamente no } \\
\text { agravamento e aumento das complicações dos } \\
\text { sintomas de pacientes com a COVID-19. }\end{array}$ \\
\hline Almeida et al., 2020. & $\begin{array}{l}\text { Os achados recentes elucidam uma associação negativa em } \\
\text { indivíduos obesos admitidos com SARS-CoV-2, especialmente } \\
\text { no que tange resposta imunológica, risco acentuado para o } \\
\text { desenvolvimento de SDRA, já que ambas partilham caminhos } \\
\text { metabólicos e inflamatórios comuns, que reverberam para } \\
\text { exacerbação da doença. }\end{array}$ & $\begin{array}{l}\text { Dessa forma, para evitar a progressão e reduzir } \\
\text { os resultados graves da COVID-19, se faz } \\
\text { necessário um monitoramento e gerenciamento } \\
\text { mais efêmero e criterioso nesse público por } \\
\text { parte dos profissionais de saúde, além disso, se } \\
\text { faz pertinente, a adoção de medidas pautadas no } \\
\text { estilo de vida saudável para melhorar tanto o } \\
\text { estado nutricional, como também marcadores } \\
\text { sistêmicos das funções imunológicas nesse } \\
\text { período pandêmico. }\end{array}$ \\
\hline Pitanga et al., 2020. & $\begin{array}{l}\text { Considerando os benefícios da prática regular de atividade } \\
\text { física para os sistemas cardiometabólico e imunológico, bem } \\
\text { como para a saúde mental, é muito importante que as pessoas } \\
\text { sejam conscientizadas para continuidade desse comportamento } \\
\text { durante o período da pandemia do COVID-19, para assim, } \\
\text { melhor suportar os efeitos de uma possível contaminação por } \\
\text { infecções virais. }\end{array}$ & $\begin{array}{l}\text { O aumento dos níveis de atividade física na } \\
\text { população pode ajudar a combater a obesidade e } \\
\text { outros distúrbios cardiometabólicos, além de } \\
\text { melhorar a saúde mental e a função } \\
\text { imunológica, e, consequentemente nos preparar } \\
\text { melhor, tanto para a atual pandemia de COVID- } \\
\text { 19, quanto para outras futuras pandemias com } \\
\text { características similares. }\end{array}$ \\
\hline Martelleto et al., 2021. & $\begin{array}{l}\text { Para dados estatísticos foram consultados conceituados sites } \\
\text { institucionais nacionais e internacionais. Sumarizando, } \\
\text { confirmou-se que a obesidade e suas comorbidades estão } \\
\text { relacionadas aos principais fatores de risco para a COVID-19. }\end{array}$ & $\begin{array}{l}\text { A inflamação sistêmica característica de ambas } \\
\text { as doenças, assim como a disfunção tecidual } \\
\text { advinda da invasão celular pelo SARS-CoV-2 } \\
\text { propiciada pelo seu receptor na membrana } \\
\text { celular, são críticos para o pior prognóstico da } \\
\text { COVID-19 em pacientes obesos. }\end{array}$ \\
\hline Bolsoni-Lopes et al., 2021. & $\begin{array}{l}\text { Este estudo traz à tona reflexões importantes para os } \\
\text { profissionais de saúde, pesquisadores e gestores, desde o } \\
\text { princípio da pandemia, período no qual a obesidade não era } \\
\text { reconhecida como fator de risco, até o cenário atual, no qual } \\
\text { uma serie de mecanismos fisiopatológicos que conectam } \\
\text { clinicamente essas doenças estão sendo propostos. }\end{array}$ & $\begin{array}{l}\text { A obesidade é um fator de risco para } \\
\text { agravamento da COVID-19, que está } \\
\text { contribuindo para o congestionamento dos } \\
\text { serviços de saúde, e que requer uma assistência } \\
\text { de saúde diferenciada, com ajustes de protocolos } \\
\text { assistenciais, farmacológicos e empenho na } \\
\text { educação em saúde no âmbito do Sistema Único } \\
\text { de Saúde. }\end{array}$ \\
\hline Silva et al., 2021. & $\begin{array}{l}\text { A seleção resultou em noveestudos, sendo que quatro estudos - } \\
44,4 \% \text { (E3, E5, E6 e E7) - apresentaram a prevalência de } \\
\text { obesidade em adultos hospitalizados por COVID-19, dois } \\
\text { estudos - 22,2\% (E6, E9) - associaram a obesidade ao } \\
\text { desenvolvimento da COVID-19 grave, três estudos - 33,3\% } \\
\text { (E1, E4 e E7) - associaram a obesidade à necessidade de } \\
\text { ventilação mecânica e três estudos - 33,3\% (E2, E4 e E8) - } \\
\text { associaram a obesidade à mortalidade por COVID-19. }\end{array}$ & $\begin{array}{l}\text { A obesidade trata-se de uma doença crônica não } \\
\text { transmissível, sendo um fator de risco } \\
\text { considerado importante para o agravamento da } \\
\text { doença COVID-19, no entanto, é passível de } \\
\text { prevenção, pois hábitos saudáveis de vida } \\
\text { podem reduzir o quadro grave de infecção por } \\
\text { COVID-19. }\end{array}$ \\
\hline
\end{tabular}




\begin{tabular}{|c|l|l|}
\hline Christinelli et al., 2021. & $\begin{array}{l}\text { Participaram 26 mulheres, emergindo a categoria temática } \\
\text { "Dificuldades e potencialidades na adesão às mudanças no } \\
\text { estilo de vida durante a pandemia". Dentre as dificuldades, } \\
\text { estão a mudança da rotina o o aumento da ansiedade; e, dentre } \\
\text { as potencialidades, estão o acompanhamento multiprofissional, } \\
\text { diminuição da tensão e controle de peso. }\end{array}$ & $\begin{array}{l}\text { A percepção dos participantes mostrou } \\
\text { fragilidades consideráveis na adesão ao } \\
\text { programa, causadas pelo distanciamento social. } \\
\text { Estudos com intervenções remotas são } \\
\text { importantes para melhorar a qualidade desse } \\
\text { tipo de assistência à saúde. }\end{array}$ \\
\hline Costa et al., 2020. & $\begin{array}{l}\text { A obesidade é definida pelo aumento do tecido adiposo no no } \\
\text { organismo de forma crônica, sendo provocada por causas } \\
\text { multifatoriais. Na infecção pelo SARSCoV-2 a faixa pediátrica } \\
\text { é associada a um melhor prognóstico e a uma baixa taxa de } \\
\text { mortalidade. }\end{array}$ & $\begin{array}{l}\text { Embora a literatura seja limitada, é possível } \\
\text { determinar a existência de uma conexão entre a } \\
\text { relação da infeç̧ão pelo SARS-CoV-2 e a } \\
\text { obesidade, podendo ser estabelecida pelo risco } \\
\text { aumentado de desenvolvimento da forma grave } \\
\text { da doença, uma vez que a obesidade torna um } \\
\text { agravante do quadro infeccioso sistêmico. }\end{array}$ \\
\hline
\end{tabular}

Fonte: SCIELO e BVS.

De acordo com os artigos científicos encontrados podemos observar que até agora, sabe-se que a propagação do SARS-CoV-2 ocorre principalmente após a inalação de partículas virais e sua entrada no sistema respiratório. O vírus tem alta afinidade pela enzima conversora da angiotensina-2 (ACE-2), que é um receptor que permite a entrada de COVID-19 e é expresso em vários órgãos, como coração, epitélio alveolar, pâncreas e tecido adiposo. Embora o tecido pulmonar seja um importante local de entrada para SARS-CoV-2, a expressão de ACE2 no tecido adiposo é mais proeminente, levando a uma maior vulnerabilidade e risco de pessoas obesas (Almeida et al., 2020; Bil, 2021).

Devido à rápida expansão da pandemia COVID-19, ficou claro que, além de outras comorbidades de risco previamente estimadas, a obesidade também está associada a sintomas mais graves, maiores taxas de hospitalização e maiores taxas de mortalidade. Esse aspecto tem sido negligenciado na descrição inicial da infecção, que ganhou bastante relevância com os dados epidemiológicos de países ocidentais, principalmente dos Estados Unidos, onde a prevalência de obesidade é de cerca de $40 \%$, muito superior à da China, que é de apenas $6 \%$. Um estudo do Reino Unido descobriu que $72 \%$ dos pacientes admitidos na UTI devido ao COVID-19 eram obesos ou com sobrepeso (Giacaglia, 2020).

Dados internacionais preocupantes mostram que a frequência de obesidade é muito alta entre pacientes gravemente enfermos que recebem tratamento intensivo de SARS-CoV-2. No Brasil, a obesidade é a principal comorbidade associada ao óbito em menores de 60 anos. Pessoas obesas têm quatro vezes mais risco de lesões e morte devido ao COVID-19 (BolsoniLopes et al., 2021).

Durante a recente pandemia de COVID-19 no Canadá, a obesidade foi considerado o terceiro fator demográfico mais prevalente entre as crianças internadas na UTI, perdendo apenas para as crianças com doenças graves, imunossupressão e câncer. Em Nova York, a obesidade é a comorbidade mais comum em 50 casos graves de COVID-19, afetando crianças e adolescentes (Nogueira de Almeida et al., 2020).

A compreensão dos profissionais de saúde sobre os mecanismos fisiopatológicos que ligam a obesidade e COVID-19 é essencial para o cuidado adequado. Para tanto, precisamos primeiro saber que além do armazenamento de energia dos mamíferos, o tecido adiposo também é o centro regulador do metabolismo corporal e o órgão endócrino produtor de hormônios, adipocinas e citocinas (Bolsoni-Lopes et al., 2021).

O tecido adiposo é geralmente considerado um tecido inerte e funcionalmente considerado um armazenamento de energia. Ele também desempenha um papel central na regulação das funções imunológicas e endócrinas da resposta inflamatória à infecção. A obesidade visceral está relacionada a distúrbios metabólicos e é considerada um fator de alto risco durante a infecção por SARS-CoV-2 (Maurya et al., 2021). 
O conceito de adipócitos, como depósitos de gordura, tornou-se uma coisa do passado. Este tecido altamente vascularizado interage com todo o corpo e expressa um grande número de enzimas, hormônios e citocinas inflamatórias (CKI). Com a expansão das células de gordura na obesidade, principalmente na área visceral, o CKI será liberado cronicamente, incluindo fator de necrose tumoral alfa (TNF-a), interleucina 1 b e 6, fator de atração de macrófagos (MCP-1), o Inibidor do ativador de plasminogênio fator (PAI-1), enzima conversora de angiotensina 1 (ACE1), resistina e leptina, etc. Portanto, a obesidade pode levar a um estado inflamatório crônico, que é determinado pela interferência direta das células gordurosas hipertróficas com a capacidade regulatória dos linfócitos T, linfócitos B e macrófagos, que favorece as vias pró-inflamatórias e estimula a produção de CKI (Giacaglia, 2020).

A principal característica fisiológica de pacientes obesos é que o tecido adiposo (TA) é abundante e extenso no corpo, sendo esse tecido um órgão de bastante importância endócrina, bem como para o metabolismo, sendo associado com o sistema imunológico e a homeostase metabólica. Portanto, o aumento da carga desses pacientes com aumento do peso levará ao desequilíbrio da resposta linfática e da medula óssea, o que irá alterar o sistema imunológico e será acompanhado pela expansão da resposta inflamatória, levando a uma maior disseminação de infecções virais, como a pandemia que ocorre pela infecção por SARS-CoV-2 (De Figueiredo et al., 2020; Perez et al., 2021).

Devido à importância do tecido adiposo nas mais diversas atividades desempenhadas pelo corpo humano, é necessário compreender sua distribuição para classificar as condições de obesidade, pois o peso por si só não pode indicar adequadamente essa distribuição. Em estudos epidemiológicos, utiliza-se o índice de massa corporal (IMC), que faz com que a relação entre peso e altura ao quadrado $(\mathrm{kg} / \mathrm{m} 2)$ classifique os indivíduos com ou não excesso de peso. O IMC é dividido em sobrepeso ou obesidade grau I (IMC 25 a 29,9 kg / m2), obesidade grau II (IMC 30 a 39,9 kg / m2) e obesidade grau III (IMC> 40 kg / m2). É importante ressaltar que outros parâmetros devem ser avaliados para que as medidas encontradas não sejam subestimadas ou superestimadas, interferindo no diagnóstico (De Figueiredo et al., 2020).

A obesidade é uma doença intimamente relacionada à piora da taxa de internação hospitalar por síndrome respiratória aguda. A deposição excessiva de adipocinas inflamatórias pode prejudicar a resposta imunológica de indivíduos infectados, prejudicar a quimiotaxia e impedir a diferenciação de macrófagos. O desequilíbrio na liberação de adipocinas pró-inflamatórias e anti-inflamatórias pode desencadear uma tempestade de citocinas que é comum em pacientes criticamente enfermos. Além disso, o tecido adiposo pode servir como reservatório para uma variedade de vírus, incluindo SARS-CoV-2, que tem a capacidade de disseminar a infecção de órgãos para tecidos adiposos adjacentes, dificultando e prolongando a eliminação do vírus (Schuh, 2021; Patel et al., 2021).

A Figura 2 demonstra associação entre obesidade e aumento de peso atuando de forma direta na gravidade e aumento das complicações dos sintomas de pacientes com a COVID-19. 
Figura 2. Fluxograma da associação entre obesidade e aumento de peso atuando de forma direta na gravidade e aumento das complicações dos sintomas de pacientes com a COVID-19, Teresina Piaú́, Brasil 2021.

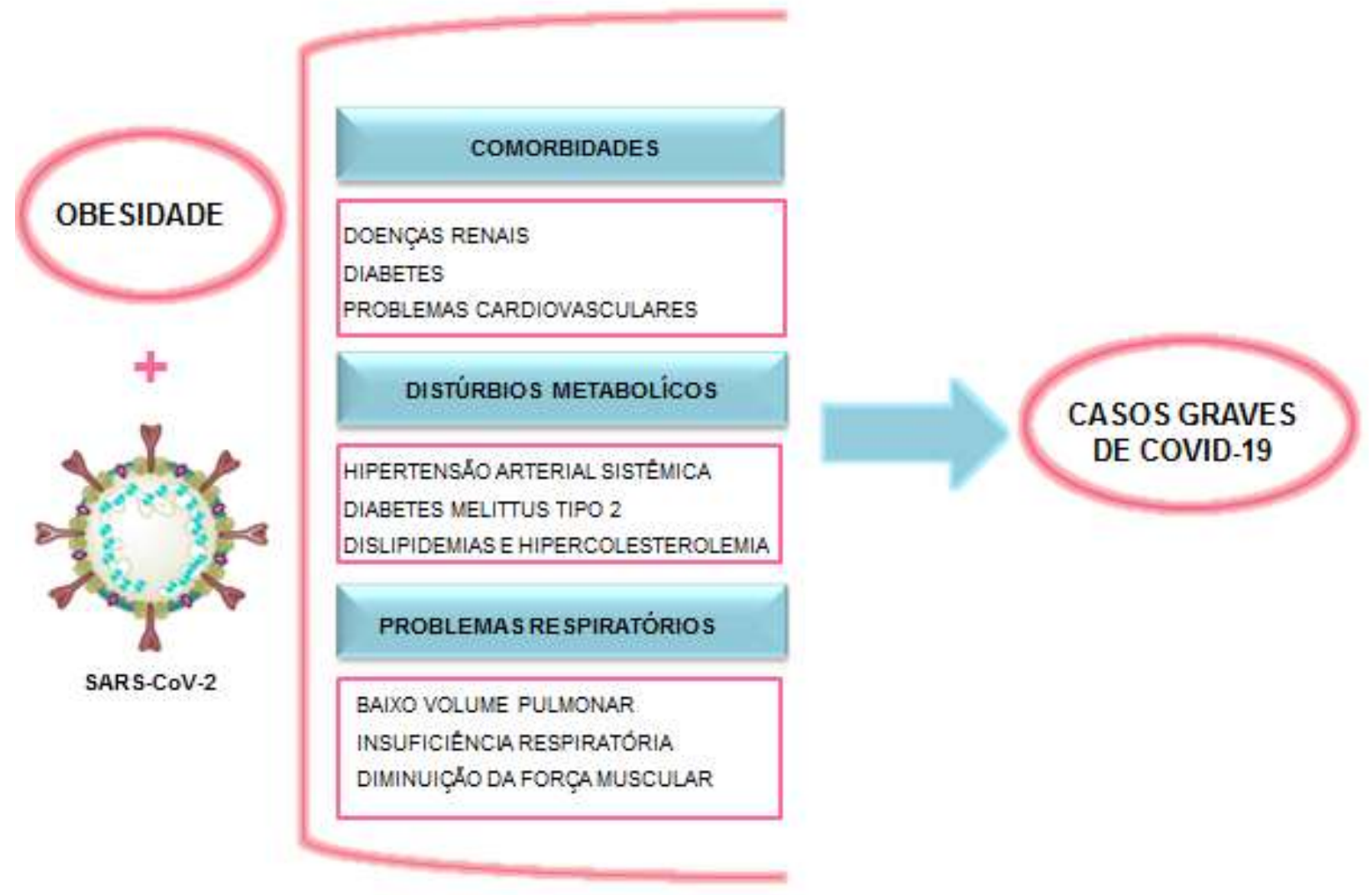

Fonte: Adaptado de De Figueiredo et al. (2020).

Pacientes obesos podem ter complicações respiratórias e estão associados a um risco aumentado de diabetes, doenças cardiovasculares e renais. Além disso, a presença de hipertensão, dislipidemia e diabetes tipo 2 pode tornar os indivíduos suscetíveis a eventos cardiovasculares e aumentar a suscetibilidade a infecções. A obesidade e o sobrepeso podem afetar diretamente o agravamento e aumento dos sintomas e complicações em pacientes com COVID-19 (De Figueiredo et al., 2020).

Muitas evidências científicas relacionam direta ou indiretamente a obesidade com a maioria dos fatores de alto risco do COVID-19. Pessoas com diabetes, hipertensão e obesidade grave têm maior probabilidade de serem infectadas e apresentarem mais complicações e possível óbito. Casos anteriores de SARS-CoV e MERS-CoV demonstraram a relação entre obesidade e casos mais graves de infecções virais. Estudos apontam também que pacientes com obesidade estão mais sujeitos à hospitalização (Martelleto et al., 2021).

A obesidade associada ao COVID-19 parece levar a um mau prognóstico em termos de dificuldade de intubação, manejo do paciente, perturbação, e em especial ao mal funcionamento das células do sistema imunológico, porque a obesidade reflete o desequilíbrio entre as citocinas pró-inflamatórias e anti-inflamatórias, e o processo inflamatório. Os principais mediadores IL-6, TNF $\alpha$, IL-8, leptina e adiponectina induzem estresse oxidativo e levam a inflamação de baixo grau, prejudicando o processo de regulação da resposta imune e inflamatória (Almeida et al., 2020; Costa et al., 2020).

A infecção grave por COVID-19 considera o sobrepeso e a obesidade como fatores de risco, que podem estar relacionados a vários mecanismos, como hiper-reatividade imunológica, resposta metabólica prejudicada e os efeitos adversos da obesidade na função pulmonar, volume expiratório forçado reduzido e capacidade vital dificultada (Silva et al., 2021). 
Pacientes obesos com infecção por SARS-CoV-2 apresentam complicações clínicas graves no caso de síndrome do desconforto respiratório agudo, lesão cardíaca, doença tromboembólica, coagulação intravascular difusa e doença pulmonar obstrutiva crônica grave. A lesão pulmonar aguda é outra causa importante de doença grave e morte em pacientes infectados com SARS-CoV-2. Outro fator importante que pode causar desequilíbrio imunológico em pacientes obesos com COVID-19 são as adipocinas superiores de longo prazo, como a leptina (Maurya et al., 2021).

Ressalta-se a importância do incentivo ao autocuidado com base na manutenção de hábitos saudáveis dos obesos, principalmente durante o período de distanciamento social causado pela COVID-19, pois a obesidade grave é uma condição clínica que pode agravar a infecção. Indivíduos obesos tem alteração em diferentes estágios das respostas imunes inatas e adaptativas, caracterizadas por condições inflamatórias crônicas e de baixo grau, que podem piorar a condição (Christinelli et al., 2021).

\section{Conclusão}

Conclui-se que a obesidade e o sobrepeso têm impacto direto na deterioração dos pacientes, com a presença de sintomas e complicações dos pacientes com COVID-19. Os principais motivos estão relacionados ao aumento dos adipócitos e das doenças relacionadas a essa patologia, tais como: distúrbios do sistema imunológico, redução das reservas respiratórias, capacidade funcional e complacência do sistema respiratório, diabetes, hipertensão e existência de doenças respiratórias crônicas.

Considerando que, como os pacientes obesos compartilham de alterações das vias metabólicas e inflamatórias devido ao acúmulo de tecido adiposo, estudos mostram que eles são mais suscetíveis a complicações graves de Covid-19, portanto, os profissionais de saúde são obrigados a realizar monitoramento e manejo mais breves e sábios para evitar a progressão e reduzir a gravidade de COVID -19 .

É necessária a realização de pesquisas utilizando protocolos clínicos e epidemiológicos para determinar a relação entre sobrepeso, obesidade e aumento do risco de complicações do COVID-19, a fim de gerar e estabelecer um entendimento mais profundo e melhor dessa associação, incluindo o entendimento das complicações concomitantes de pacientes com sobrepeso. Vale ressaltar também a importância de um estilo de vida saudável, incluindo alimentação balanceada, exercícios físicos regulares, conscientização da população quanto ao distanciamento social e hábitos de higiene adequados para evitar a contaminação e disseminação do vírus.

\section{Referências}

Almeida, P. T, Teixeira, Y., Lopes, L. S, Sobreira, T. X, da Cruz Santana, E. N, da Silva, C. J. R. S, \& Bispo, R. S. (2020). Preditores de riscos advindos da obesidade que repercutem em mau prognóstico em pacientes infectados por Sars-CoV-2. Research, Society and Development, 9 (9), e929997961e929997961.

Bil, J., \& Możeńska, O. (2021). The vicious cycle: a history of obesity and COVID-19. BMC Cardiovascular Disorders, 21(1), 1-5.

Bolsoni-Lopes, A., Furieri, L. B., \& Alonso-Vale, M. I. C. (2021). Obesidade e a Covid-19: uma reflexão sobre a relação entre as pandemias. Revista Gaúcha de Enfermagem, 42.

Christinelli, H. C. B., Spigolon, D. N., Teston, E. F., Costa, M. A. R., Westphal, G., Nardo, N., \& Fernandes, C. A. M. (2021). Percepções de adultos com obesidade sobre monitoramento remoto multiprofissional no início da pandemia de COVID-19. Revista Brasileira de Enfermagem, 74.

Costa, L. R., de Oliveira Mueller, M. E., Frauches, J. P., Campos, N. B., de Oliveira, L. S., Gentilin, K. F.., \& Mello, P. (2020). Obesidade infantil e quarentena: crianças obesas possuem maior risco para a COVID-19. Revista Residencia Pediátrica.

De Figueiredo, M. C. F., do Nascimento, J. M. F., Araújo, D. S., Silva, T. R., Barros, F. D. D., de Moura, F. V. P., \& Pereira-Freire, J. A. (2020). O impacto do excesso de peso nas complicações clínicas causadas pela COVID-19: Uma revisão sistemática. Research, Society and Development, 9(7), e693974791e693974791.

Giacaglia, L. R. (2020). Covid-19, obesidade e resistência à insulina. Ulakes Journal Of Medicine, 1, 2-10. 
Maurya, R., Sebastian, P., Namdeo, M., Devender, M., \& Gertler, A. (2021). Gravidade do COVID-19 na obesidade: interação da leptina e das citocinas inflamatórias na relação entre a alta morbidade e a mortalidade. Frontiers in Immunology , 12, 2349.

Martelleto, G. K. S., Alberti, C. G., Bonow, N. E., Giacomini, G. M., Neves, J. K., de Miranda, E. C. A., \& de Macedo, I. C. (2021). Principais fatores de risco apresentados por pacientes obesos acometidos de COVID-19: uma breve revisão. Brazilian Journal of Development, 7(2), 13438-13458.

Nogueira-de-Almeida, C. A., Del Ciampo, L. A., Ferraz, I. S., Del Ciampo, I. R., Contini, A. A., \& Ued, F. D. V. (2020). COVID-19 and obesity in childhood and adolescence: a clinical review. Jornal de Pediatria, 96, 546-558.

Patel, K.H, Li, X., Quint, J.K, Ware, J.S, Peters, N.S, \& Ng, F.S (2021). O aumento da adiposidade e a presença de morbidade cardiometabólica estão associados ao aumento da mortalidade relacionada a Covid-19: resultados do UK Biobank. BMC Endocrine Disorders , 21 (1), 1-6.

Perez, A., Naljayan, M., Shuja, I., Florea, A., \& Reisin, E. (2021). Hypertension, Obesity, and COVID-19: a Collision of Pandemics. Current Hypertension Reports, 23(6), 1-7.

Pitanga, F. J. G., Beck, C. C., \& Pitanga, C. P. S. (2020). Inatividade física, obesidade e COVID-19: perspectivas entre múltiplas pandemias. Revisto Brasileira de Atividade Física \& Saúde, 25, 1-4.

Schuh, L. C. (2021). O impacto da obesidade no agravamento e mortalidade por Covid-19: evidências de revisões sistemáticas e metanálises

Silva, G. M. D., Pesce, G. B., Martins, D. C., Carreira, L., Fernandes, C. A. M., \& Jacques, A. E. (2021). Obesidade como fator agravante da COVID-19 em adultos hospitalizados: revisão integrativa. Acta Paulista de Enfermagem, 34. 